\title{
Comparison of severe and critical COVID-19 patients imported from Russia with and without influenza A infection in Heilongjiang Province: a retrospective study
}

\author{
Qingqing Dai ${ }^{1 \#}$, Ming Ye ${ }^{1 \#}$, Zhiqiang Tang ${ }^{1}$, Kaijiang Yu ${ }^{2}$, Yang Gao ${ }^{2}$, Zhenyu Yang ${ }^{1}$, Junbo Zheng ${ }^{1}$, \\ Shu Zuo ${ }^{1}$, Yan Liu ${ }^{3}$, Fengjie Xie ${ }^{4}$, Qiuyuan Han ${ }^{1}$, Hua He ${ }^{5}$, Hongliang Wang ${ }^{1}$ \\ ${ }^{1}$ Department of Critical Care Medicine, the Second Affiliated Hospital of Harbin Medical University, Harbin, China; ${ }^{2}$ Department of Critical Care \\ Medicine, the First Affiliated Hospital of Harbin Medical University, Harbin, China; ${ }^{3}$ Department of Medical Statistics, Harbin Medical University, \\ Harbin, China; ${ }^{4}$ Department of Critical Care Medicine, Hongqi Hospital of Mudanjiang Medical University, Mudanjiang, China; ${ }^{5}$ Department of \\ Infectious Disease, Hongqi Hospital of Mudanjiang Medical University, Mudanjiang, China \\ Contributions: (I) Conception and design: Q Dai, H Wang; (II) Administrative support: M Ye, H Wang, H He; (III) Provision of study materials or \\ patients: K Yu, Z Tang; (IV) Collection and assembly of data: H He, Y Gao, Z Yang; (V) Data analysis and interpretation: Q Dai, J Zheng, S Zuo, Y \\ Liu, F Xie, Q Han; (VI) Manuscript writing: All authors; (VII) Final approval of manuscript: All authors. \\ \#These authors contributed equally to this work. \\ Correspondence to: Hua He. Department of Infectious Disease, Hongqi Hospital of Mudanjiang Medical University, No. 5, Tongxiang Road, Aimin \\ District, Mudanjiang 157011, China. Email: hehua.6187079@163.com; Hongliang Wang. Department of Critical Care Medicine, the Second \\ Affiliated Hospital of Harbin Medical University, 246 Xuefu Road, Harbin 150086, China. Email: icuwanghongliang@163.com.
}

Background: The rapid spread of coronavirus disease-19 (COVID-19) poses a global health emergency, and cases entering China from Russia are quite diverse. This study explored and compared the clinical characteristics and outcomes of severe and critically ill COVID-19 patients from Russia with and without influenza A infection, treated in a northern Chinese hospital (Russia imported patients).

Methods: A total of 32 severe and critically ill Russia-imported COVID-19 patients treated in the Heilongjiang Imported Severe and Critical COVID-19 Treatment Center from April 6 to May 11, 2020 were included, including 8 cases (group A) with and 24 cases (group B) without influenza A infection. The clinical characteristics of each group were compared, including prolonged hospital stay, duration of oxygen therapy, time from onset to a negative SARS-CoV-2 qRT-PCR RNA $\left(\mathrm{T}_{\text {neg }}\right)$ result, and duration of bacterial infection.

Results: The results showed that blood group, $\mathrm{PaO}_{2} / \mathrm{FiO}_{2}$, prothrombin time (PT), prothrombin activity (PTA), computed tomography (CT) score, hospital stay, duration of oxygenation therapy, $\mathrm{T}_{\text {neg }}$, and duration of bacterial infection were statistically different between the two groups $(\mathrm{P}<0.05)$. Multivariant regression analysis showed that the Sequential Organ Failure Assessment (SOFA) score, C-reactive protein (CRP), and influenza A infection were factors influencing hospital stay; SOFA score, CRP, and CT score were factors influencing the duration of oxygenation therapy; $\mathrm{PaO}_{2} / \mathrm{FiO}_{2}$, platelet count (PLT), and CRP were factors influencing $\mathrm{T}_{\text {neg }}$; and gender, SOFA score, and influenza A infection were factors influencing the duration of bacterial infection.

Conclusions: Influenza A infection is common in Russia-imported COVID-19 patients, which can prolong the hospital stay and duration of bacterial infection. Routinely screening and treating influenza A should be conducted early in such patients.

Keywords: Coronavirus disease-19 influenza A (COVID-19 influenza A); imported patients; clinical characteristics; clinical outcome

Submitted Jul 19, 2021. Accepted for publication Aug 26, 2021.

doi: 10.21037/atm-21-3912

View this article at: https://dx.doi.org/10.21037/atm-21-3912 


\section{Introduction}

The outbreak of the coronavirus disease-19 (COVID-19), previously known as $2019-\mathrm{nCoV}$, was initially reported in December $2019(1,2)$, and rapidly spread, creating a global health emergency. Zhou et al. (3) demonstrated that the over-expression of angiotensin converting enzyme 2 (ACE2) from different species in HeLa cells with human ACE2, pig ACE2, civet ACE2, (but not mouse ACE2) allowed SARS-CoV-2 infection and replication, thereby showing SARS-CoV-2 uses ACE2 as a cellular entry receptor. Until now, there have been no specific vaccines or therapeutics available, and the current management of COVID-19 includes travel restriction, patient isolation, and supportive medical care. Therefore, a better understanding of the underlying pathology of COVID-19 is required.

By the end of March 2020, the peak of the current outbreak of COVID-19 in China was over, as new domestic cases kept declining and the overall epidemic situation remained at a low level. At the time, it was believed that the epidemic could be brought under control by June. However, since April 6, 2020 there has been a burst of Russia-imported patients crossing through the Suifenhe border, resulting in the immediate establishment of the Heilongjiang Imported Severe and Critical COVID-19 Treatment Center. People receiving primary screening at the border were transferred to the center, and due to their travelling history, the clinical characteristics of this are population differ the general population. Surprisingly, we noted that some COVID-19 patients were also infected with influenza $\mathrm{A}$.

Influenza A virus subtype H1N1 (A/H1N1) was the most common cause of human influenza (flu) in 2009. In April of that year, an outbreak of influenza-like illness (ILI) occurred in Mexico then spread to the United States (4), and the rest of the world (5). Pandemic influenza viruses often cause severe disease in middle-aged adults without pre-existent co-morbidities (6), with several inflammatory mediators significantly up-regulated in peripheral and lung samples from $\mathrm{A} / \mathrm{H} 1 \mathrm{~N} 1$-infected patients who develop severe pneumonia (7). Influenza A infection is also accompanied by a characteristic impairment of the innate immune responses (8). How the complication of influenza A infection affect the clinical characteristics and outcome of COVID-19 patients is yet to be confirmed.

In the current study, the clinical characteristics of 32 severe and critically ill patients diagnosed with COVID-19 are discussed. Influenza A infection is potentially a factor that affects the severity and prognosis of COVID-19 patients, which has not been clearly elucidated before. To our knowledge, this is the first study to elucidate the differences between COVID-19 patients with and without influenza $\mathrm{A}$ infection in an imported cohort. Our findings may provide further details on the epidemic and the clinical characteristics of this novel coronavirus, may assist the establishment of further effective measures to control the disease. We present the following article in accordance with the STROBE reporting checklist (available at https://dx.doi. org/10.21037/atm-21-3912).

\section{Methods}

\section{Study design and participants}

A total of 409 COVID-19 patients were imported from Russia from April 6, 2020 until the time or writing. All patients were classified into four clinical types; mild, moderate, severe, and critical, based on the Diagnosis and Treatment of New Coronavirus Pneumonia (seventh edition) from the National Health Commission of the People's Republic of China (9). Thirty-two patients treated in the Heilongjiang Imported Severe and Critical COVID-19 Treatment Center from April 6 to May 11, 2020 were included in this study, and throat swab samples from the upper respiratory tract for COVID-19 and influenza A virus nucleic acid test (RT-PCR assay) were obtained from all patients at admission. Exclusion criteria were as follows: Patients with age $<18$ years old; pregnant women; patients who deceased within 24 hours after admission. Eight COVID-19 patients with influenza A infection were included in group A and 24 without influenza A infection were included in group B. The study was conducted in accordance with the Declaration of Helsinki (as revised in 2013). And the study was approved by the Research Ethics Committee of the Second Affiliated Hospital of Harbin Medical University (KY2020-174). Individual consent for this retrospective analysis was waived.

\section{Data collection}

Data including demographic data, exposure history, underlying comorbidities, blood group, body temperature, vital signs, laboratory findings, chest computed tomography (CT) scans, and treatment measures such as antiviral therapy, corticosteroid treatment, or respiratory support, 
were recorded. The Sequential Organ Failure Assessment (SOFA) score, and Acute Physiology and Chronic Health Evaluation II (APACHE II) scores were determined on the day of ICU admission.

\section{CT score}

Two radiologists reviewed all chest CT images and determined the score by consensus, and were kept blinded to COVID-19 RT-PCR results. Each of the five lung lobes (two at left and three at right) was assessed, and the percentage of lobar involvement classified as none ( $0 \%)$, minimal (1-25\%), mild $(26-50 \%)$, moderate $(51-75 \%)$, or severe $(76-100 \%)$, with a corresponding score of $0,1,2,3$, or 4 , respectively. The total severity score (TSS) was calculated by summing the five lobe scores (range from 0 to 20) (10).

\section{COVID-19 treatment}

Patients with $\mathrm{PaO}_{2} / \mathrm{FiO}_{2}<300 \mathrm{mmHg}$ or shortness of breath were offered oxygen therapy including invasive mechanical ventilation (IMV), non-invasive ventilation (NIV), high-flow nasal cannula oxygenation (HFNC), and normal nasal cannula accordingly. Patients with no contraindications were offered a combination of $200 \mathrm{mg}$ of oral hydroxychloroquine and Lianhuaqingwen capsules. Patients with influenza A infection were offered $150 \mathrm{mg}$ of oseltamivir, 3 times per day for 10 days, and for those with secondary bacterial pneumonia, a broad spectrum anti-biotic (levofloxacin) was added. All patients received intravenous administration of corticosteroid with a dosage of $1-2 \mathrm{mg} / \mathrm{kg} / \mathrm{d}$ for $5-7$ days depending on their severity.

\section{Outcome indicators}

The primary outcome we chose was the length of hospital stay, as all patients survived. Patients with body temperature restored to normal for more than 3 days, significantly improved respiratory symptoms, significant improvement on chest imaging, and two successive negative PCR assays of COVID-19 virus nucleic acid test (interval $\geq 24$ hours) were discharged (9). The secondary outcome indicators were duration of oxygen therapy, time from onset to a negative SARS-CoV-2 qRT-PCR RNA $\left(\mathrm{T}_{\text {neg }}\right)$, and duration of bacterial infection. Secondary bacterial infection was diagnosed when patients showed clinical infection symptoms, signs of pneumonia or bacteraemia, elevated white blood cell (WBC) counts, neutrophil parentage (NEUT\%), C-reactive protein (CRP), or procalcitonin (PCT), as well as positive CT and sputum culture results (11).

\section{Statistical analysis}

All statistical analyses were performed using Statistical Analysis System (SAS) (version 9.4, SAS Institute Inc., Cary, NC, USA). Quantitative data which were normally distributed are presented as mean \pm standard deviation $(\bar{x} \pm s)$, and data which were not normally distributed are presented as median and interquartile range (IQR) $[M$ (P25, P75)]. Qualitative data are presented as case number (percentage). In comparison of quantitative data, the $t$-test was carried out if the data were normally distributed and met homogeneity variance, and the $t^{\prime}$-test was carried out if the data were normally distributed and did not meet homogeneity variance. If the data were not normally distributed, comparisons were performed with the Wilcoxon rank-sum test, and the comparisons of qualitative data were performed with the Fisher's exact test (because the total case number was $32<40$ ). $\mathrm{P}<0.05$ was considered as statistically significant.

The influence of factors including hospital stay, duration of oxygen therapy, $T_{\text {neg }}$, and duration of bacterial infection were analyzed with linear regression. Factors with statistical difference in univariant regression analysis were included in a multivariant regression model, and backward selection was performed to screen them (significance level for entry $<0.05$, significance level for stay $<0.10$ ).

\section{Results}

\section{General characteristics}

Altogether, 409 Russia-imported patients attended our hospital, and 32 with severe and critical COVID-19 were clustered and provided a history of exposure at the border. Most were Chinese businessmen in Russia and most received timely diagnosis and treatment as the government formulated an efficient early warning and isolation program. Group A ( $\mathrm{n}=8)$ was composed of six men and two women with a mean age of $48.63 \pm 10.08$ years, and in group B $(n=24)$, there were 14 men and 10 women, with a mean age of $46 \pm 8.75$ years (Table 1). Approximately $28.1 \%$ (9/32) patients in our cohort required advanced ventilatory support and rescue therapies for profound hypoxemic respiratory failure, including HFNC, high levels of inspired oxygen and 


\section{Page 4 of 12}

Dai et al. COVID-19 patients with and without influenza A infection

Table 1 Comparison of different parameters between two groups

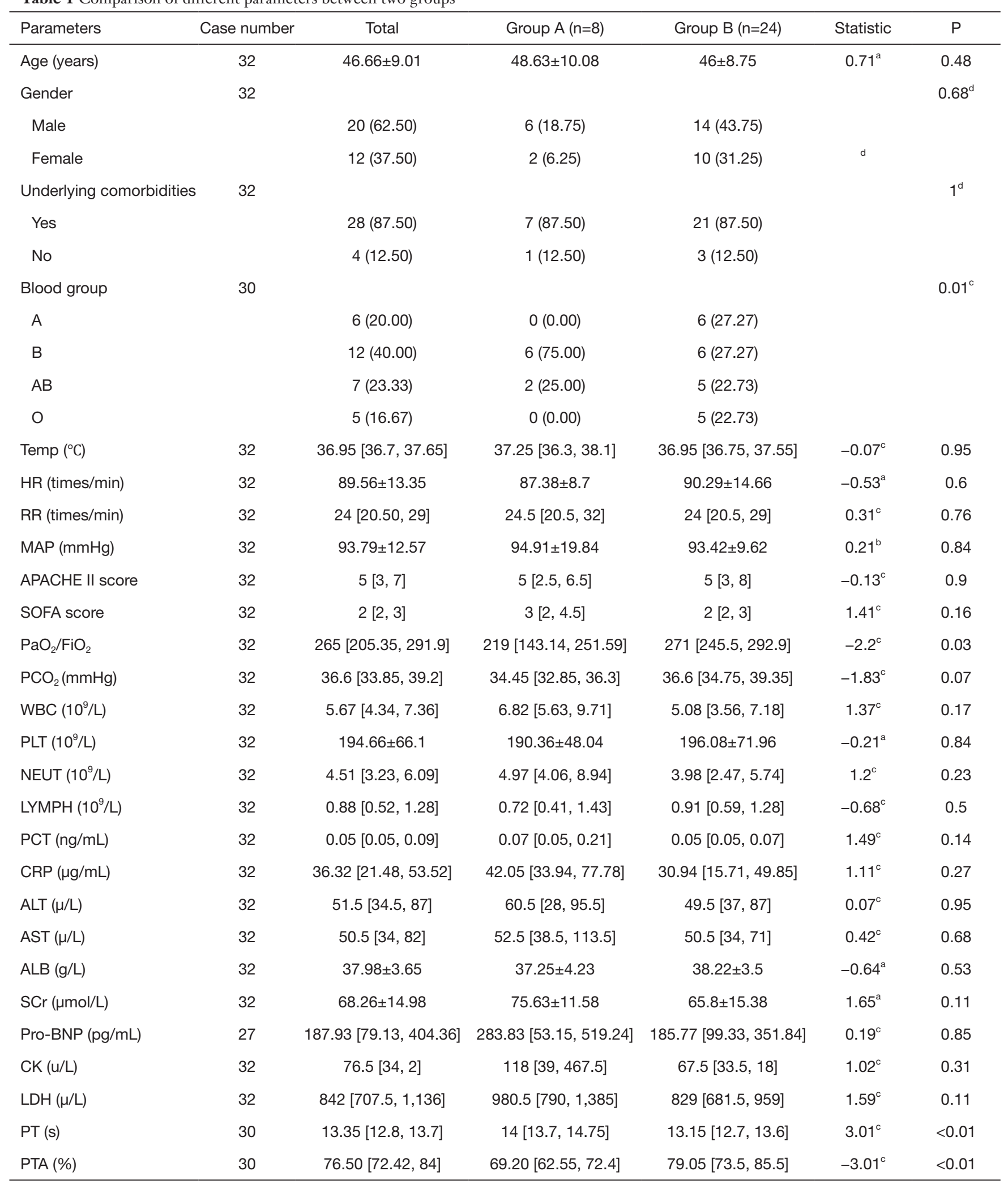

Table 1 (continued) 
Table 1 (continued)

\begin{tabular}{|c|c|c|c|c|c|c|}
\hline Parameters & Case number & Total & Group A $(n=8)$ & Group B $(n=24)$ & Statistic & $\mathrm{P}$ \\
\hline $\mathrm{DD}(\mu \mathrm{g} / \mathrm{mL})$ & 29 & $1.04[0.89,1.26]$ & $1.21[0.94,1.72]$ & $1.04[0.89,1.19]$ & $1.33^{\mathrm{c}}$ & 0.19 \\
\hline AT III (\%) & 30 & $61.55[52.3,81.1]$ & $66.6[46.3,81.7]$ & $61.55[52.3,80.1]$ & $-0.12^{\mathrm{C}}$ & 0.91 \\
\hline CT score & 32 & $8[5,16]$ & $20[12.5,20]$ & $7[5,10]$ & $3.27^{\mathrm{c}}$ & $<0.01$ \\
\hline No & & 11 [34.38] & 1 [3.13] & $10[31.25]$ & & \\
\hline Yes & & 21 [65.63] & 7 [21.88] & 14 [43.75] & & \\
\hline Hospital stay (days) & 32 & $21[14.5,23.5]$ & $24.5[23,25]$ & $19.5[10.5,22]$ & $2.89^{c}$ & $<0.01$ \\
\hline $\begin{array}{l}\text { Duration of oxygenation } \\
\text { therapy (days) }\end{array}$ & 32 & $14.31 \pm 7.55$ & $20.50 \pm 7.15$ & $12.25 \pm 6.6$ & $3^{a}$ & $<0.01$ \\
\hline
\end{tabular}

${ }^{a}$, represents the data are normally distributed and meet homogeneity variance; the comparison was performed with the $t$-test; ${ }^{b}$, represents the data are normally distributed and do not meet homogeneity variance; the comparison was performed with the $t^{\prime}$-test; ' , represents the data are not normally distributed; the comparison was performed with the Wilcoxon rank-sum test; ${ }^{d}$, represents the comparison was performed with the Fisher's exact test and no statistics were available. Temp, temperature; HR, heart rate; RR, respiratory rate; MAP, mean arterial pressure; APACHE II, Acute Physiology and Chronic Health Evaluation II; SOFA, Sequential Organ Failure Assessment; $\mathrm{PCO}_{2}$, pressure of carbon dioxide; WBC, white blood cell count; PLT, platelet count; NEUT, neutrophil count; LYMPH, lymphocyte count; PCT, procalcitonin; CRP, C-reactive protein; ALT, alanine transaminase; AST, aspartate aminotransferase; ALB, Albumin; $\mathrm{SCr}$, serum creatinine; pro-BNP, pro B-type natriuretic peptide; CK, creatine kinase; LDH, lactate dehydrogenase; PT, prothrombin time; PTA, prothrombin activity; APTT, activated partial thromboplastin time; DD, D-dimer; AT III, antithrombin III; CT score, computed tomography score; $T_{\text {neg }}$, time from onset to a negative SARS-CoV-2 qRT-PCR RNA.
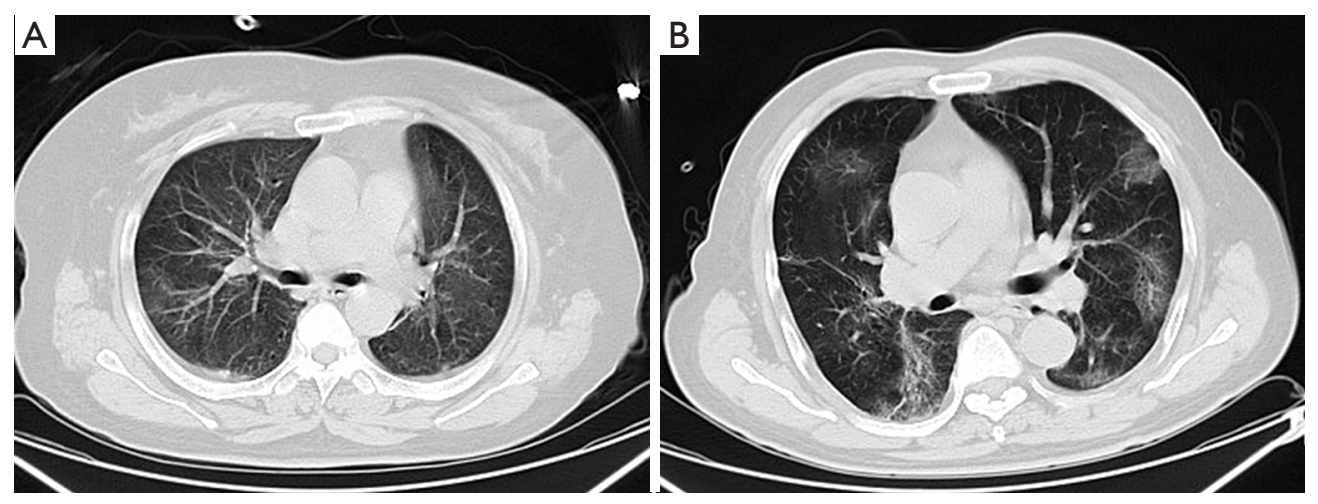

Figure 1 Typical characteristics of CT in two groups in the early stage. (A) Typical CT image of group A with presence of diffuse bilateral GGOs and obscure boundary. (B) Typical CT image of group B, multifocal GGOs with clear lesion margin can be observed in bilateral lungs. Subpleural distribution was common. CT, computed tomography; GGOs, ground-glass opacities.

positive end expiratory pressure (PEEP), pressure control, prone positioning ventilation, and neuromuscular blockade. No patient required extracorporeal membrane oxygenation.
The typical CT characteristic of simple COVID-19 and those complicated with influenza $\mathrm{A}$ at admission are shown in Figure 1. 
Table 2 Factors influencing hospital stay

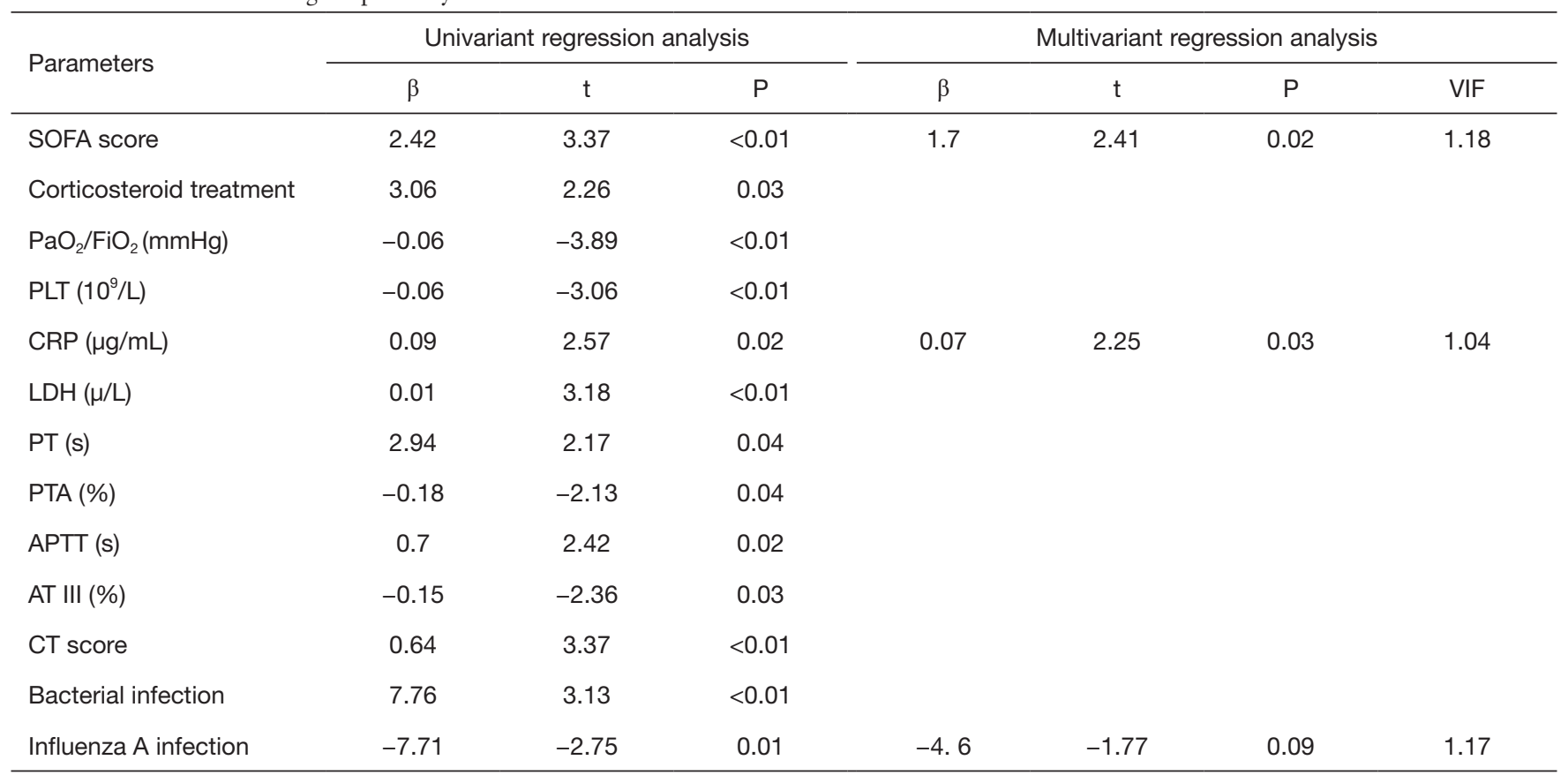

After the analysis was adjusted by age and gender, the factors with statistical significance in multivariant analysis were not changed. VIF, variance inflation factor; SOFA, Sequential Organ Failure Assessment; PLT, platelet count; CRP, C-reactive protein; LDH, lactate dehydrogenase; PT, prothrombin time; PTA, prothrombin activity; APTT, activated partial thromboplastin time; AT III, antithrombin III; CT score, computed tomography score.

\section{Comparisons of characteristics between two groups}

Comparisons of different parameters between the two groups showed that blood group, $\mathrm{PaO}_{2} / \mathrm{FiO}_{2}$, prothrombin time (PT), prothrombin activity (PTA), CT score, hospital stay, duration of oxygenation therapy, $\mathrm{T}_{\text {neg }}$, and duration of bacterial infection were statistically different $(\mathrm{P}<0.05)$. The PT, CT score, hospital stay, duration of oxygenation therapy, $\mathrm{T}_{\text {neg }}$, and duration of bacterial infection were higher in group A than group $\mathrm{B}$, while $\mathrm{PTA}$ and $\mathrm{PaO}_{2} / \mathrm{FiO}_{2}$ in group A were lower than group B. Group A contained only patients with blood group $B$ and $A B$, and the number of patients with blood group B was significantly larger than that in group B, as shown in Table 1 .

\section{Factors influencing hospital stay}

Univariant linear regression analysis showed that the SOFA score, corticosteroid treatment, $\mathrm{PaO}_{2} / \mathrm{FiO}_{2}$, platelet count (PLT), CRP, lactate dehydrogenase (LDH), PT, PTA, activated partial thromboplastin time (APTT), antithrombin III (AT III), CT score, bacterial infection, and influenza A infection were factors influencing hospital stay $(\mathrm{P}<0.05)$.

Backward multivariant linear regression analysis was then performed to screen the final influencing factors, with $R^{2}$ as 0.4492 and adjusted $\mathrm{R}^{2}$ as 0.3901 . The results showed that higher SOFA score, higher CRP, and influenza A infection were risk factors for prolonged hospital stay $\left(\mathrm{P}_{\text {entry }}<0.05\right.$, $\mathrm{P}_{\text {stay }}<0.1$ ), as shown in Table 2 .

\section{Factors influencing duration of oxygenation therapy}

Univariant linear regression analysis showed that the SOFA sore, $\mathrm{PaO}_{2} / \mathrm{FiO}_{2}$, CRP, serum creatinine ( $\mathrm{SCr}$ ), creatine kinase (CK), LDH, PT, PTA, APTT, AT III, CT score, bacterial infection, and influenza A infection were factors that influenced the duration of oxygenation therapy $(\mathrm{P}<0.05)$.

Backward multivariant linear regression analysis was then performed to screen the final influential factors, where $\mathrm{R}^{2}$ was 0.5390 and adjusted $R^{2}$ was 0.4896 . The results showed that higher SOFA score, higher CRP, and higher CT score were risk factors for the prolonged duration of oxygenation 
Table 3 Factors influencing duration of oxygenation therapy

\begin{tabular}{|c|c|c|c|c|c|c|c|}
\hline Parameters & \multicolumn{3}{|c|}{ Univariant regression analysis } & \multicolumn{4}{|c|}{ Multivariant regression analysis } \\
\hline SOFA score & 2.58 & 3.68 & $<0.01$ & 1.51 & 2.22 & 0.04 & 1.32 \\
\hline $\mathrm{PaO}_{2} / \mathrm{FiO}_{2}$ & -0.07 & -4.76 & $<0.01$ & & & & \\
\hline $\mathrm{CRP}(\mu \mathrm{g} / \mathrm{mL})$ & 0.1 & 2.83 & 0.01 & 0.07 & 2.36 & 0.03 & 1.07 \\
\hline CK $(\mu / L)$ & 0.01 & 2.56 & 0.02 & & & & \\
\hline $\mathrm{LDH}(\mu / \mathrm{L})$ & 0.01 & 3.23 & $<0.01$ & & & & \\
\hline PT (s) & 3.89 & 2.84 & 0.01 & & & & \\
\hline PTA (\%) & -0.21 & -2.36 & 0.03 & & & & \\
\hline CT score & 0.72 & 4.05 & $<0.01$ & 0.44 & 2.4 & 0.02 & 1.36 \\
\hline Bacterial infection & 7.82 & 3.16 & $<0.01$ & & & & \\
\hline Influenza A infection & -8.25 & -3 & $<0.01$ & & & & \\
\hline
\end{tabular}

After the analysis was adjusted by age and gender, the factors with statistical significance in multivariant analysis were not changed. VIF, Variance inflation factor; SOFA, Sequential Organ Failure Assessment; CRP, C-reactive protein; SCr, serum creatinine; CK, creatine kinase; LDH, lactate dehydrogenase; PT, prothrombin time; PTA, prothrombin activity; APTT, activated partial thromboplastin time; AT III, antithrombin III; CT score, computed tomography score.

therapy $\left(\mathrm{P}_{\text {entry }}<0.05, \mathrm{P}_{\text {stay }}<0.1\right)$, as shown in Table 3 .

\section{Factors influencing $T_{\text {neg }}$}

Univariant linear regression analysis results showed that $\mathrm{PaO}_{2} / \mathrm{FiO}_{2}$, PLT, CRP, LDH, APTT, CT score, and bacterial infection were factors influencing $\mathrm{T}_{\text {neg }}(\mathrm{P}<0.05)$.

Backward multivariant linear regression analysis was then performed to screen the final influential factors, where $\mathrm{R}^{2}$ was 0.4676 and adjusted $R^{2}$ was 0.4105 . The results showed that lower $\mathrm{PaO}_{2} / \mathrm{FiO}_{2}$, lower PLT, and higher CRP were risk factors for prolonged $\mathrm{T}_{\text {neg }}\left(\mathrm{P}_{\text {entry }}<0.05, \mathrm{P}_{\text {stay }}<0.1\right)$, as shown in Table 4.

\section{Factors influencing duration of bacterial infection}

Univariant linear regression analysis showed that gender, body temperature, APACHE II score, SOFA score, corticosteroid treatment, $\mathrm{PaO}_{2} / \mathrm{FiO}_{2}$, SCr, pro B-type natriuretic peptide (pro-BNP), LDH, PT, PTA, CT score, bacterial infection, and influenza $\mathrm{A}$ infection were factors influencing the duration of bacterial infection $(\mathrm{P}<0.05)$.
Backward multivariant linear regression analysis was then performed to screen the final influence factors, where $\mathrm{R}^{2}$ was 0.7111 and adjusted $\mathrm{R}^{2}$ was 0.6912 . The results showed that male gender, higher SOFA score, and influenza A infection were risk factors for prolonging the duration of bacterial infection $\left(\mathrm{P}_{\text {entry }}<0.05, \mathrm{P}_{\text {stay }}<0.1\right)$, as shown in Table 5 .

\section{Discussion}

In this study we compared the clinical characteristics and outcomes of severe and critical COVID-19 patients with and without influenza A infection, and the results showed that the proportion of influenza A infection in Russiaimported severe and critical COVID-19 patients was up to $25 \%(8 / 32)$. Due to different seasonal characteristics in the northern and southern parts of China, the annual influenza A epidemic manifests various characteristics. As reported, the flu epidemic in the north of China usually begins in October and ends in March of the following year, peaking in January and February (12). Patterns in the north of China are relevant for the epidemic characteristics of the population and are important in determining effective 
Table 4 Factors influencing $\mathrm{T}_{\text {neg }}$

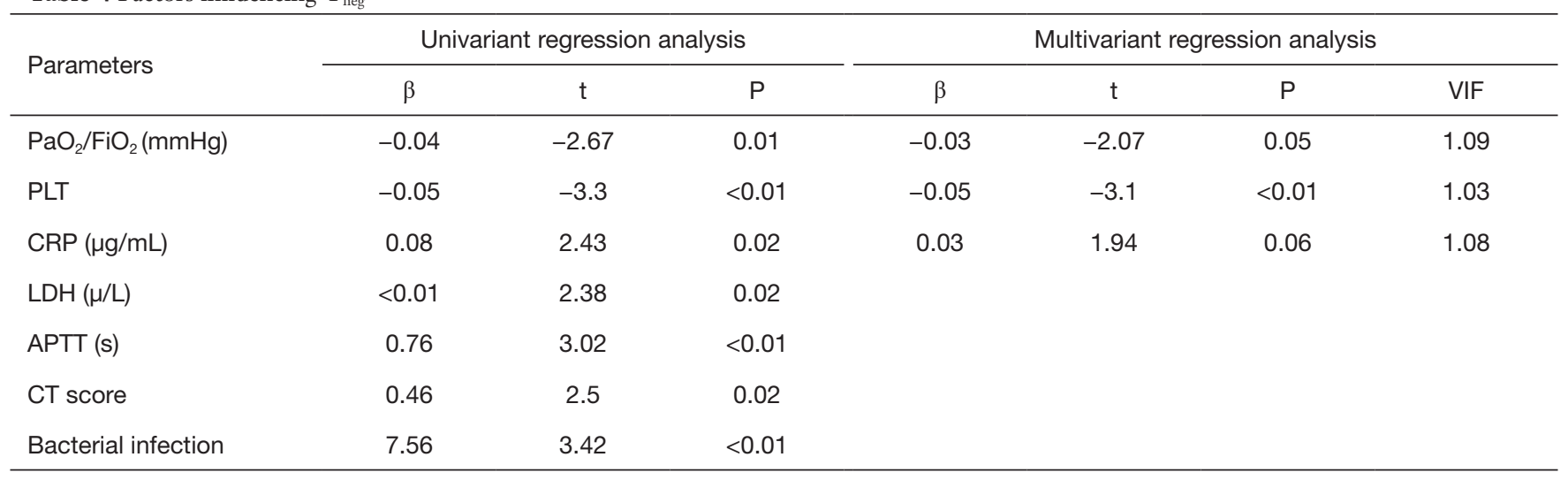

After the analysis was adjusted by age and gender, the factors with statistical significance in multivariant analysis were not changed. $T_{\text {neg, }}$ time from onset to a negative SARS-CoV-2 qRT-PCR RNA; VIF, variance inflation factor; PLT, platelet count; CRP, C-reactive protein; LDH, lactate dehydrogenase; APTT, activated partial thromboplastin time; CT score, computed tomography score.

Table 5 Factors influencing duration of bacterial infection

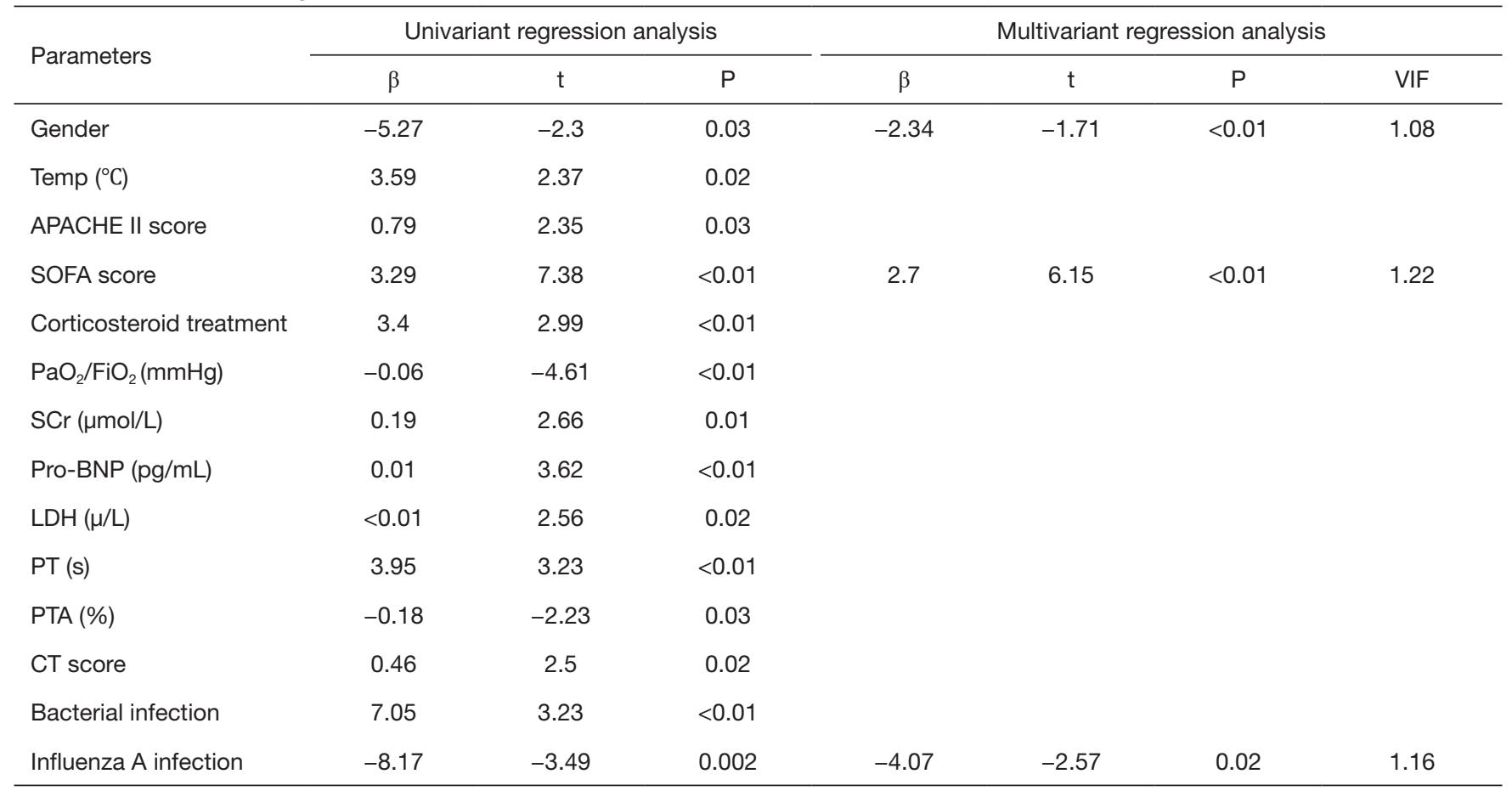

After the analysis was adjusted by age and gender, gender was a factor influencing the duration of bacterial infection. The duration of bacterial infection in females was shorter than in males. VIF, Variance inflation factor; Temp, temperature; APACHE II, Acute Physiology and Chronic Health Evaluation II; SOFA, Sequential Organ Failure Assessment; SCr, serum creatinine; pro-BNP, pro B-type natriuretic peptide; LDH, lactate dehydrogenase; PT, prothrombin time; PTA, prothrombin activity; CT score, computed tomography score.

control measures. Compared with SARS and H1N1 influenza, COVID-19 is more contagious, more concealed in transmission, greater infectious intensity and more severe in clinical manifestations. In addition, the immune function of COVID-19 patients is poor, due to the reduction in CD4+ and CD8+ T lymphocytes $(13,14)$. Therefore, patients travelling from Russia to China face a high risk of being infected with influenza, and screening for the 
disease and taking control measures in advance to cut off its transmission are the key to controlling the pandemic.

The infected patients were relatively young (46.66 9.01 years), and compared with patients from Wuhan, the $\mathrm{T}_{\text {neg }}\left(16.78 \pm 6.89\right.$ days) was shorter (15). $\mathrm{PaO}_{2} / \mathrm{FiO}_{2}$, PLT, CRP, LDH, APTT, CT score, and bacterial infection were factors influencing $T_{\text {neg }}$, which may be a early warning to predict the isolation duration thus to provide the best isolation and treatment strategies. The ratio of severe and critical cases was also much lower $(7.82 \%, 32$ severe and critical cases/409 total imported cases) due to the younger age of patients, with less underlying comorbidities and good immune status. Patients responded positively to the treatment, which strengthened their confidence to fight COVID-19. The involvement of multi-organs was not common in severe and critical patients with a SOFA score of $5.41 \pm 3.28$, and injuries in this cohort mainly targeted the lung. However, COVID-19 patients in Wuhan were usually complicated with multi-organ injury such as cardiac injury, shock, and kidney injury $(16,17)$. In Wuhan patients, the complication of influenza A caused deterioration, and the symptoms of respiratory failure were significantly aggravated.

Influenza A infection in COVID-19 patients prolonged outcome indicators including the hospital stay, duration of oxygenation therapy, $\mathrm{T}_{\text {neg }}$, and duration of bacterial infection. In addition, $\mathrm{PaO}_{2} / \mathrm{FiO}_{2}$, and the CT score in COVID-19 patients with influenza A were worse, suggesting the lung was the main target organ and further affected the outcome indicators. This was further verified by CT manifestations. The clinicopathological features of influenza pneumonia include bronchial and surrounding alveolar hyperemia, inflammatory exudation, and formation of a transparent membrane (18), contributing to a clustered ground-glass opacity (GGO). However, CT of COVID-19 patients showed multifocal GGOs (Figure 1A). Based on the typical CT characteristics at onset, this study showed that the CT feature of group A held great value distinguishing influenza A patients in the early stage. It is also worthy to mention that as reported in previous studies, $\mathrm{H} 1 \mathrm{~N} 1$ is one of the independent risk factors for pulmonary embolism. H1N1 ARDS patients had a 23.3-fold higher risk for pulmonary embolism and could even suffer disseminated intravascular coagulation $(19,20)$. The prolonged PT and decreased PTA in group A suggest that influenza A infection related coagulopathy might increase the risk of poor outcome.

It is interesting to note that the ratio of patients with blood type B was much higher in group A than group B (75\% vs. $27.27 \%)$. It was previously reported that people with blood group A have a higher risk while people with blood group O have a lower risk for SARS-Cov-2 infection and COVID-19 severity $(2,21,22)$. The relation between $\mathrm{ABO}$ blood group and influenza $\mathrm{A}$ infection has also been previously reported. An observation of influenza patients showed that the degree of immune response after influenza A (H1N1), A (H3N2), and B viruses differed significantly in subjects with different blood groups of the $\mathrm{ABO}(\mathrm{H})$ system (23). The combination of the virus and red blood cells may be related to blood group glyco-proteins or mediated by molecules that appear more frequently on type B red blood cells (24). Collectively, these results suggest the $A B O$ system may provide an opportunity for establishing a biomarker for differential susceptibility of influenza A in COVID-19 patients. A larger cohort study should be conducted in the future to verify the confluence and the combination mode of pandemic virus and receptor.

Influenza A infection is a risk factor for a prolonged duration of hospital stay and duration of bacterial infection. COVID-19 patients have been shown to display a complex immune dysfunction that could render them susceptible to early bacterial co-infection (25). While prolonged bacterial infection in group A may have significantly prolonged the hospital stay, Influenza A was not a risk factor for the duration of oxygenation therapy. While univariant regression analysis showed influenza A infection was significant, in multivariate regression analysis it was less critical compared with the SOFA score, CRP, and CT score. However, the proportion of mechanical ventilation was much higher in group A $(25 \%, 2 / 8)$ than group B $(4.17 \%$, $1 / 24)$, and the proportion of HFNC was also significantly higher in group A $(75 \%, 6 / 8)$ than group B $(12.5 \%, 3 / 24)$. The large proportion of patients requiring advanced respiratory support illustrates that influenza A infection could significantly affect advanced ventilator support but was not the key factor affecting the total duration of oxygenation therapy. The results also showed influenza A infection was not a risk factor for prolonged $T_{\text {neg }}$ in COVID-19 patients. Although influenza A and COVID-19 can both affect the immune function of patients $(8,13,26)$, complicating with influenza A might not affect the clearance of novel coronavirus. The underlying mechanism remains to be elucidated.

The characteristics of some very common coronavirus laboratory tests are of great significance for its clinical diagnosis and treatment. As reported previously, LDH 
and CRP should be considered as useful tests for the early identification of patients who require tighter respiratory monitoring and more active supportive therapy to avoid poor prognosis (27). It was also reported that lymphocytes, CRP, PCT, alanine transaminase (ALT), aspartate aminotransferase (AST), LDH, D-dimer, CD4 T cells, and interleukin (IL)-6 provide valuable signals for preventing the deterioration of COVID-19 patients (28). We also screened some common and user-friendly parameters including CRP and PLT as risk factor for outcome indexes, which were supported by the above studies.

While in China, the COVID-19 epidemic is largely under control, the global pandemic remains. Early screening of influenza A and the early intervention and prevention of secondary bacterial infection will be critical in treating imported cases. Once typical CT scanning is performed, physicians should be highly alerted to the possibility of influenza A infection, and all patients with the disease should be given oseltamivir in the early stage. Oseltamivir exerts its antiviral activity by inhibiting the activity of the viral neuraminidase enzyme on the surface of the influenza viruses $A$, which prevents budding from the host cell, viral replication, and infectivity (29). In a 5-year analysis of oseltamivir timing and clinical outcomes, early administration after hospital admission was associated with shortened hospitalization (30). However, as the coronavirus does not carry neuraminidase enzyme, oseltamivir has no effect on COVID-19. As a treatment strategy, we propose the early screening for influenza A. in COVID-19 patients, and once confirmed, propose early oseltamivir treatment to prevent them developing severe respiratory failure. By now, the government are highly alerted about COVID-19 and influenza A infection. Early screening, effective isolation, vaccine and advanced rescue measures are implemented to improve the prognosis of COVID-19 patients.

There are some limitations to this study, the first of which is its retrospective nature. Secondly, although we have included all severe and critical patients at the border during this epidemic, the population is isolated, and the sample size is relatively small. Thirdly, the interaction between COVID-19 and H1N1 remains unclear, and further study is needed to explore the underlying mechanism.

In conclusion, influenza A infection is common in imported severe and critically ill COVID-19 patients from Russia. Influenza A infection can prolong the hospital stay and duration of bacterial infection, which may lead to prognosis deterioration and increase the economic burden. Early routine screening and oseltamivir treatment can be used as a preventive measure in imported patients.

\section{Acknowledgments}

We acknowledge all health-care workers, technical and paramedical staff, and laboratories involved in the diagnosis and treatment of patients at Heilongjiang Imported Severe and Critical COVID-19 Treatment Center in this difficult context. We also thank two radiologists: Chenglei Zhao (The Second Affiliated Hospital of Harbin Medical University, Harbin, China) and Hongwei Wang (The Second Affiliated Hospital of Harbin Medical University, Harbin, China) for reviewing the $\mathrm{CT}$ results.

Funding: This work was supported by the International Medical Exchange Foundation of China (grant number Z-2018-35-1902), National Natural Science Foundation of China (grant number 82002077), and Nn10 Program of Harbin Medical University Cancer Hospintal.

\section{Footnote}

Reporting Checklist: The authors have completed the STROBE reporting checklist. Available at https://dx.doi. org/10.21037/atm-21-3912

Data Sharing Statement: Available at https://dx.doi. org/10.21037/atm-21-3912

Conflicts of Interest: All authors have completed the ICMJE uniform disclosure form (available at https://dx.doi. org/10.21037/atm-21-3912). The authors have no conflicts of interest to declare.

Ethical Statement: The authors are accountable for all aspects of the work in ensuring that questions related to the accuracy or integrity of any part of the work are appropriately investigated and resolved. All procedures performed in this study involving human participants were in accordance with the Declaration of Helsinki (as revised in 2013). The study was approved by Research Ethics Committee of the Second Affiliated Hospital of Harbin Medical University (No.: KY2020-174). Individual consent for this retrospective analysis was waived.

Open Access Statement: This is an Open Access article distributed in accordance with the Creative Commons Attribution-NonCommercial-NoDerivs 4.0 International License (CC BY-NC-ND 4.0), which permits the non- 
commercial replication and distribution of the article with the strict proviso that no changes or edits are made and the original work is properly cited (including links to both the formal publication through the relevant DOI and the license). See: https://creativecommons.org/licenses/by-nc-nd/4.0/.

\section{References}

1. Chen L, Liu W, Zhang Q, et al. RNA based mNGS approach identifies a novel human coronavirus from two individual pneumonia cases in 2019 Wuhan outbreak. Emerg Microbes Infect 2020;9:313-9.

2. Lu R, Zhao X, Li J, et al. Genomic characterisation and epidemiology of 2019 novel coronavirus: implications for virus origins and receptor binding. Lancet 2020;395:565-74.

3. Zhou P, Yang XL, Wang XG, et al. A pneumonia outbreak associated with a new coronavirus of probable bat origin. Nature 2020;579:270-3.

4. Cameron MJ, Rowe T, Kelvin DJ. Possible link between the severe respiratory illness outbreak in Mexico and swine influenza in southwestern United States? J Infect Dev Ctries 2009;3:157-8.

5. Smith GJ, Vijaykrishna D, Bahl J, et al. Origins and evolutionary genomics of the 2009 swine-origin H1N1 influenza A epidemic. Nature 2009;459:1122-5.

6. Monsalvo AC, Batalle JP, Lopez MF, et al. Severe pandemic $2009 \mathrm{H} 1 \mathrm{~N} 1$ influenza disease due to pathogenic immune complexes. Nat Med 2011;17:195-9.

7. Zúñiga J, Torres $M$, Romo J, et al. Inflammatory profiles in severe pneumonia associated with the pandemic influenza A/H1N1 virus isolated in Mexico City. Autoimmunity 2011;44:562-70.

8. Giamarellos-Bourboulis EJ, Raftogiannis M, Antonopoulou A, et al. Effect of the novel influenza A (H1N1) virus in the human immune system. PLoS One 2009;4:e8393.

9. Diagnosis and Treatment Protocol for Novel Coronavirus Pneumonia (Trial Version 7). Chin Med J (Engl) 2020;133:1087-95.

10. Chung M, Bernheim A, Mei X, et al. CT imaging features of 2019 novel coronavirus (2019-nCoV). Radiology 2020;295:202-7.

11. Zhou Y, Guo S, He Y, et al. COVID-19 is distinct from SARS-CoV-2-negative community-acquired pneumonia. Front Cell Infect Microbiol 2020;10:322.

12. Shu YL, Fang LQ, de Vlas SJ, et al. Dual seasonal patterns for influenza, China. Emerg Infect Dis
2010;16:725-6.

13. De Biasi S, Meschiari M, Gibellini L, et al. Marked T cell activation, senescence, exhaustion and skewing towards TH17 in patients with COVID-19 pneumonia. Nat Commun 2020;11:3434.

14. Huang I, Pranata R. Lymphopenia in severe coronavirus disease-2019 (COVID-19): systematic review and metaanalysis. J Intensive Care 2020;8:36.

15. Li Q, Cao Y, Chen L, et al. Hematological features of persons with COVID-19. Leukemia 2020;34:2163-72.

16. Wang D, Hu B, Hu C, et al. Clinical characteristics of 138 hospitalized patients with 2019 novel coronavirus-infected pneumonia in Wuhan, China. JAMA 2020;323:1061-9. Erratum in: JAMA 2021;325:1113.

17. Huang C, Wang Y, Li X, et al. Clinical features of patients infected with 2019 novel coronavirus in Wuhan, China. Lancet 2020;395:497-506.

18. Fujita J, Ohtsuki Y, Higa H, et al. Clinicopathological findings of four cases of pure influenza virus A pneumonia. Intern Med 2014;53:1333-42.

19. Goeijenbier M, van Gorp EC, Van den Brand JM, et al. Activation of coagulation and tissue fibrin deposition in experimental influenza in ferrets. BMC Microbiol 2014;14:134.

20. Keller TT, van der Sluijs KF, de Kruif MD, et al. Effects on coagulation and fibrinolysis induced by influenza in mice with a reduced capacity to generate activated protein $\mathrm{C}$ and a deficiency in plasminogen activator inhibitor type 1. Circ Res 2006;99:1261-9.

21. Cheng Y, Cheng G, Chui CH, et al. ABO blood group and susceptibility to severe acute respiratory syndrome. JAMA 2005;293:1450-1.

22. Wu Y, Feng Z, Li P, et al. Relationship between ABO blood group distribution and clinical characteristics in patients with COVID-19. Clin Chim Acta 2020;509:220-3.

23. Sominina AA, Tsubalova LM, Karpova LS, et al. Genetic predisposition to latent influenza A virus in children with blood type B(III) as a possible cause of new epidemiologic strains in the countries of South-Eastern Asia. Vestn Ross Akad Med Nauk 1994;(9):21-4.

24. Lanteri MC, Lee TH, Wen L, et al. West Nile virus nucleic acid persistence in whole blood months after clearance in plasma: implication for transfusion and transplantation safety. Transfusion 2014;54:3232-41.

25. Kreitmann L, Monard C, Dauwalder O, et al. Early bacterial co-infection in ARDS related to COVID-19. Intensive Care Med 2020;46:1787-9. 


\section{Page 12 of 12}

26. Giamarellos-Bourboulis EJ, Netea MG, Rovina N, et al. Complex immune dysregulation in COVID-19 patients with severe respiratory failure. Cell Host Microbe 2020;27:992-1000.e3.

27. Wang F, Hou H, Wang T, et al. Establishing a model for predicting the outcome of COVID-19 based on combination of laboratory tests. Travel Med Infect Dis 2020;36:101782.

28. Bao J, Li C, Zhang K, et al. Comparative analysis of laboratory indexes of severe and non-severe patients infected with COVID-19. Clin Chim Acta

Cite this article as: Dai Q, Ye M, Tang Z, Yu K, Gao Y, Yang Z, Zheng J, Zuo S, Liu Y, Xie F, Han Q, He H, Wang H. Comparison of severe and critical COVID-19 patients imported from Russia with and without influenza A infection in Heilongjiang Province: a retrospective study. Ann Transl Med 2021;9(18):1446. doi: 10.21037/atm-21-3912

\section{Dai et al. COVID-19 patients with and without influenza A infection}

2020;509:180-94.

29. Li IW, Hung IF, To KK, et al. The natural viral load profile of patients with pandemic 2009 influenza $\mathrm{A}(\mathrm{H} 1 \mathrm{~N} 1)$ and the effect of oseltamivir treatment. Chest 2010;137:759-68.

30. Katzen J, Kohn R, Houk JL, et al. Early oseltamivir after hospital admission is associated with shortened hospitalization: a 5-year analysis of oseltamivir timing and clinical outcomes. Clin Infect Dis 2019;69:52-8.

(English Language Editor: B. Draper) 